\title{
MODELOS DE ECUACIONES DE ESTIMACIÓN GENERALIZADAS PARA EL ESTUDIO DE LA EVOLUCIÓN DE PACIENTES CON INFARTO AGUDO DE MIOCARDIO
}

\section{Erwin Kraenau Espinal y Ofelia Roque Paredes}

\section{Resumen}

Objetivo: Utilizar los modelos GEE (Generalized Estimating Equations) para explicar los indicadores de evolución de los pacientes con IAM (Infarto Agudo de Miocardio). Materiales y Métodos: La información se obtuvo de los pacientes del Hospital Dos de Mayo que estaban activos con IAM durante el año 2012. Resultados: Los mejores predictores para explicar el comportamiento del segmento ST son el sexo con un $\mathrm{OR}=0.367$ y otro diagnóstico adicional al IAM con $\mathrm{OR}=2.272$, cuando la estructura de la correlación es independiente y $\mathrm{OR}=2.359$ si la estructura de la correlación es AR(1). El predictor sexo resultó ser el único significativo para explicar la evolución de la onda T cuando la estructura de la correlación es intercambiable con un $\mathrm{OR}=9.89$.

Palabras clave: Ecuaciones de Estimación Generalizadas; Infarto Agudo de Miocardio; electrocardiograma; estructura de la correlación; derivaciones precordiales.

\section{Abstract}

Objective: To use the GEE models (Generalized Estimating Equations) to explain the indicators of progress of patients with AMI (Acute Myocardial Infarction). Materials and Methods: Information was obtained from patients of Dos de Mayo Hospital with AMI who were active during 2012. Results: The best predictors to explain the behavior of the ST segment are sex with an OR $=0.367$ and an additional diagnosis to AMI with an $\mathrm{OR}=2.272$ when the correlation 
structure is independent and $\mathrm{OR}=2.359$ if the correlation structure is $\mathrm{AR}(1)$. The predictor sex turned out to be the only significant to explain the evolution of the $\mathrm{T}$ wave when the correlation structure is interchangeable with an $\mathrm{OR}=9.89$.

Keywords: Generalized Estimating Equations; Acute Myocardial Infarction; electrocardiogram; correlation structure; precordial leads.

\section{INTRODUCCIÓN}

La enfermedad cardiovascular constituye la primera causa de mortalidad y discapacidad a nivel mundial, la mayoría de casos debidos al IAM. En el Perú, según el Instituto Nacional de Estadística (INEI), en el año 2000 las enfermedades del aparato circulatorio se encontraban entre las primeras causas de muerte $(18,2 \%$ de las muertes registradas), siendo la cardiopatía isquémica la de mayor incidencia. En el Hospital Militar Central se encontró que $14 \%$ presentó IAM Q, de ellos, el $100 \%$ fueron varones. Otro estudio en el INCOR evidenció que los pacientes con IMA Q/SICA STE, 97\% eran varones, y en la UCI coronaria del Hospital Loayza, el 75,5\% con SICA STE fueron varones.

Los hallazgos confirman que los más afectados son del género masculino (Carrión, Becerra, Pinto, \& Postigo, 2007). Las investigaciones realizadas en el Perú se han limitado a obtener la morbilidad y mortalidad, las características demográficas, epidemiológicas y clinicas de los pacientes que sufrieron IAM con el segmento ST elevado. Los pocos estudios publicados son descriptivos, por lo que se hace necesario encontrar los factores que expliquen mejor la evolución de los pacien- tes que sufrieron IAM en nuestro país a partir de la información registrada en los ECG. Se propone aplicar los modelos GEE, dada las limitaciones que presentan los modelos lineales para analizar datos longitudinales, puesto que no consideran la posible existencia de correlación entre las observaciones de cada sujeto. En los modelos lineales se ignora dicha correlación, por lo que el cálculo de la varianza de los estimadores es incorrecto y, por tanto, se llegarian a conclusiones erróneas respecto a la significación estadística. Los datos considerados en esta investigación son observaciones recogidas en diferentes momentos y referidas a la evolución del individuo que ha sufrido IAM.

\section{MÉTODOS}

\section{Población en estudio}

Se obtuvieron las historias clínicas de la Oficina de Estadística del Hospital Dos de Mayo, de los pacientes activos con IAM durante el año 2012. De cada historia se tomaron fotos del contenido de estas y principalmente de los electrocardiogramas de cada paciente (ver Figura 1). Todo esto fue recolectado durante el mes de enero del año 2013. De cada historia se extrajo información del paciente, como el 
sexo, edad, diagnóstico, y si tenía alguna otra patología, así como las medicinas consumidas después de ocurrido el evento y los electrocardiogramas.

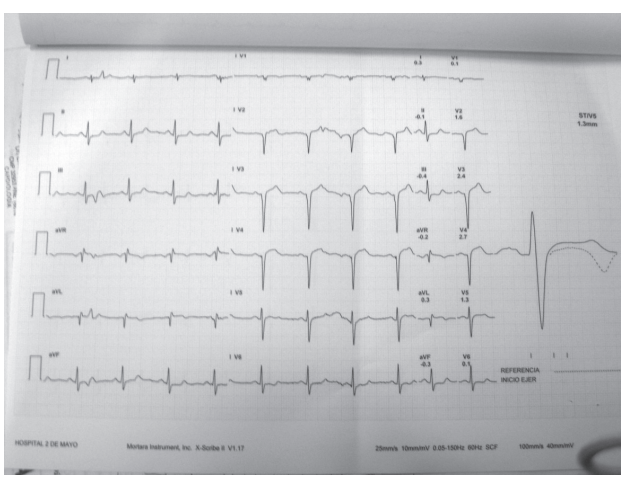

Figura 1. Fotografia del ECG de un paciente.

\section{Digitalización del ECG}

De cada historia clinica se transformó los electrocardiogramas a una forma analógica y a una forma cualitativa. El obstáculo principal es que muchos electrocardiogramas no tenían la fecha en que fueron registrados, por lo que después de hacer una depuración exhaustiva, finalmente se obtuvo la información completa de 15 pacientes diagnosticados con IAM. De cada paciente se extrajo los resultados de tres electrocardiogramas consecutivos, así como sus respectivas fechas. De cada ECG solo se utilizó la derivación precordial V2, ya que es donde se puede apreciar mejor los indicadores de evolución del paciente con IAM (Bermúdez, 1998).

Los datos fueron procesados en el software SPSS, versión 19.

\section{Elementos de electrocardiografia}

Los trazos electrocardiográficos se realizan sobre un papel donde apa- recen lineas verticales y horizontales separadas entre sí una distancia de 1 $\mathrm{mm}$. A este papel se le denomina papel electrocardiográfico, como se muestra en la Figura 2. Cada $5 \mathrm{~mm}$. las líneas son más gruesas. A lo largo de las líneas horizontales se hace la medición del tiempo; cada milimetro equivale a 0.04 seg., y el espacio que hay entre cada una de las líneas gruesas equivale a $0.20 \mathrm{seg}$. Las lineas verticales se utilizan para medir el voltaje, esto es, la separación entre cada línea horizontal equivale a $0.1 \mathrm{mV}$. La velocidad normal del papel electrocardiográfico que se utiliza es de $25 \mathrm{~mm}$. por segundo.

Las componentes de los trazos electrocardiográficos se pueden resumir como sigue:

Se denomina Onda a toda deflexión, ya sea positiva o negativa. Es positiva cuando sobrepasa hacia arriba la linea isoeléctrica, y negativa si dicha linea es sobrepasada hacia abajo. Las principales en un ECG, son:

- Onda P: Su deflexión es positiva, además de ser lenta, y esta se produce antes de que ocurra el complejo QRS. Esta onda es causada por la despolarización auricular.

- Onda Q (q): Al efectuarse la despolarización ventricular, se produce como consecuencia la onda $Q$ (q) que es una deflexión negativa inicial. Esta es anterior a la primera deflexión positiva (R).

- Onda S (s): Cuando se realiza la despolarización ventricular, se produce la primera deflexión 
negativa $\mathrm{u}$ onda $\mathrm{S}$ (s). Esta se produce después de la primera deflexión positiva $(\mathrm{R})$.

- Onda R' (r'): En el proceso de despolarización ventricular que sigue a la onda $\mathrm{S}$, se produce la segunda deflexión positiva, esto es la onda R' (r').

- Onda T: Es positiva, lenta, y sigue al complejo QRS. Esta representa la repolarización ventricular.

- Onda U: Es aquella que continúa después de la onda T. Es positiva, lenta, aparece en el intervalo TP y se produce por efecto de los pos potenciales. Generalmente indica la presencia de hipokalemia.

- Complejo QRS: Se le llama así porque es la resultante de tres ondas rápidas: la Q que antecede a $\mathrm{R}$ y es la primera negativa; la $\mathrm{R}$ es la única positiva; y la $\mathrm{S}$ es la onda negativa que sigue a la $\mathrm{R}$. Las ondas $\mathrm{P}$ y $\mathrm{T}$ representan la activación de las aurículas y la repolarización ventricular, respectivamente. El cambio de la morfología de estas ondas o disminución del tamaño en una misma derivación en un mismo pacientes, deben considerarse anormales.

En los complejos electrocardiográficos normales, las ondas que son relativamente grandes se denotan por letras mayúsculas (Q, R, S). En el papel electrocardiográficos, son aquellas que su altura es mayor de $5 \mathrm{~mm}$.; de forma análoga, las ondas relativa- mente pequeñas se denotan por letras minúsculas (q, r, s); estas son las menores de $5 \mathrm{~mm}$ (Jenkins \& Gerred, 2012).

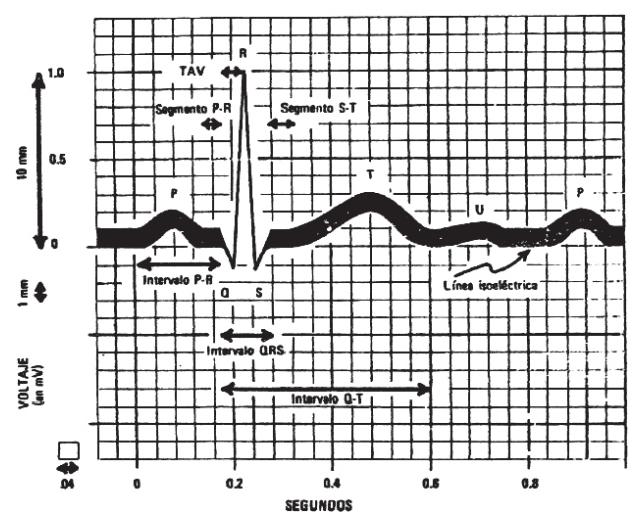

Figura 2. Electrocardiograma normal.

Un Segmento se define así al espacio comprendido entre dos ondas. Estos son:

- Segmento P-R: Es aquel que está comprendido entre el final de la onda $P$ hasta el principio del complejo QRS. En el final de este segmento está efectuándose la repolarización auricular.

- Segmento S-T: Es el tiempo transcurrido entre el punto (unión) $\mathrm{J}$, que es el final de la onda $\mathrm{S}$, hasta el principio de la onda T. Está intimamente relacionada con la repolarización ventricular y con fenómenos metabólicos, ya sean normales o anormales que suceden en el ventrículo izquierdo.

\section{Ecuaciones de Estimación Generali- zadas (GEE)}

Fueron desarrolladas por Liang y Zeger (Liang \& Zeger, 1986) como una 
extensión de los modelos de cuasi-verosimilitud, para modelar respuestas correlacionadas entre sí; más específicamente para estudios en los cuales se toma más de una medición para un mismo individuo, como los diseños longitudinales, anidados o de medidas repetidas. De esta forma, tienen dos utilidades básicas, en primer lugar, permite la estimación de los parámetros de la regresión $(\beta)$ y, en segundo lugar, proporciona la estimación simultánea de dichos parámetros y de la estructura de covarianza de la información.

Sea $\mathbf{Y}_{\mathrm{i}}=\left(Y_{i 1}, \ldots, Y_{1 t i}\right)^{t}$ el vector de respuestas multivariado del $i$-ésimo individuo, $i=1, \ldots, n$, se supone conocida la relación funcional entre la media y la varianza de cada $Y_{i j}$ :

$$
E\left(Y_{i j}\right)=\mu_{i j} \text { (a) } \quad V\left(Y_{i j}\right)=\varphi^{-1} V\left(\mu_{i j}\right)
$$

De igual forma, se asume que los individuos entre sí son independientes y que cada respuesta en un tiempo determinado, $Y_{i j}$, está asociada a un vector de variables respuestas, $x_{i j}=\left(x_{i j 1}, \ldots, x_{1 j p}\right)^{t}$ de la forma:

$$
g\left(\mu_{i j}\right)=\eta_{i j}=x_{i j}^{t} \beta
$$

Sea $R_{i}$ la matriz de correlación de las observaciones del $i$-ésimo individuo. En el caso de datos no correlacionados $R_{i}=I_{t i}$, así la ecuación (1.b) quedaría de la forma:

$$
V\left(Y_{i}\right)=\varphi^{-1} V_{i}=\varphi^{-1} \quad V_{i}^{1 / 2} R_{i} V_{i}^{1 / 2}
$$

Liang y Zeger (Liang \& Zeger, 1986) proponen estimar cada matriz de correlación como una función de un vector de parámetros, $\rho=\left(\rho_{1}, \ldots, \rho_{q}\right)^{t}$, que no depende de $\beta$. Suponiendo que todos los individuos del estudio tienen el mismo número de mediciones $\left(t_{i}=t\right)$, la matriz de varianza-covarianza de $Y_{i}$ está dada por:

$$
\Omega_{i}=\varphi^{-1} V_{i}^{1 / 2} R(\rho) V_{i}^{1 / 2}
$$

donde $R(\rho)$ es la llamada matriz de trabajo.

Para estimar $\beta$ se resuelve el sistema:

$$
\sum_{(=11)}^{n} D_{i}^{t} \Omega_{i}^{-1}\left(y_{i}-\mu_{i}\right)=0
$$

cuya solución se obtiene mediante una modificación del método iterativo de Scoring de Fisher (Agresti, 2007):

$\beta^{(m+1)}=\beta^{(m)}+\left\{\sum_{i=1}^{n} D_{i}^{(m)^{t}} \Omega_{i}^{(m)^{-1}} D_{i}^{(m)}\right\}^{-1}\left\{\sum_{(i=1)}^{n} D_{i}^{(m)^{t}} \Omega_{i}^{(m)^{-1}}\right.$ $\left.\left(y_{i}-\mu_{i}^{(m)}\right)\right\}$

$\mathrm{m}=0,1,2, \ldots$ hasta lograr la convergencia.

Las estimaciones de $\varphi$ y $\rho$ se obtienen por separado igualmente de forma iterativa. Si dichos estimadores son consistentes, $\sqrt{ } n(\hat{\beta}-\beta)$ tiende a una distribución normal cuando el tamaño de muestra es grande (Agresti, 2007).

Para el caso de respuestas dicotómicas, las ecuaciones en (1) están dadas por:

$$
E\left(Y_{i j}\right)=\pi_{i j}, \quad V\left(Y_{i j}\right)=\varphi^{-1} V\left(\pi_{i j}\right)
$$

y la función de enlace, $g\left(\pi_{i j}\right)$ puede ser cualquiera de la teoría de los Modelos Lineales Generalizados (MLG) (Bobashev \& Anthony, 2000).

\section{RESULTADOS}

Se tomó como variable respuesta la evolución del segmento ST y como predictores al diagnóstico, otro diagnóstico, sexo y edad del paciente con IAM. Se procesaron los datos teniendo en cuenta las distintas matrices de 
trabajo; en primer lugar tomando la de independencia; en segundo lugar la $\mathrm{AR}(1)$; luego la intercambiable; $y$, por último, la matriz de trabajo sin estructura. Los resultados significativos se muestran a continuación:

\section{Tabla 1}

Estimación de parámetros con tipo de respuesta logística ordinal, función de enlace logit acumulado, con modelo aditivo de efectos principales y con distintas estructuras de correlaciones tomando como variable respuesta al Segmento ST

\begin{tabular}{|c|c|c|c|c|c|c|c|c|c|c|c|}
\hline \multirow{2}{*}{ 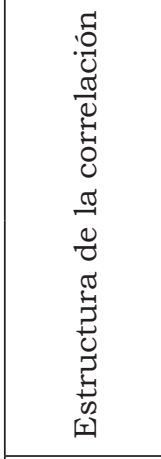 } & \multirow{2}{*}{ 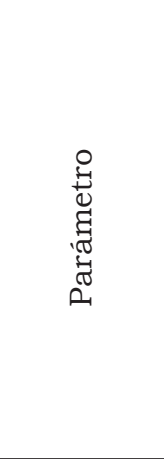 } & \multirow{2}{*}{ m } & \multirow{2}{*}{ 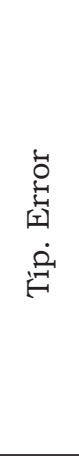 } & \multicolumn{2}{|c|}{ 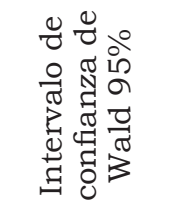 } & \multicolumn{3}{|c|}{ 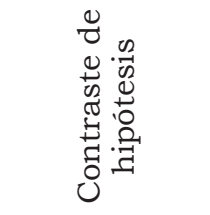 } & \multirow{2}{*}{ 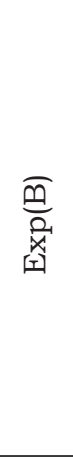 } & \multicolumn{2}{|c|}{ 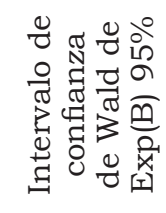 } \\
\hline & & & & 芯 & 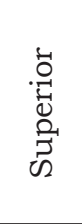 & 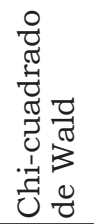 & $\vec{\sigma}$ & $\dot{\ddot{0}}$ & & . & 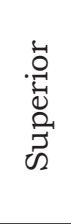 \\
\hline \multirow{2}{*}{ Independiente } & {$[\mathrm{SEXO}=1]$} & -1.004 & 0.287 & -1.566 & -0.441 & 12.228 & 1 & 0 & 0.367 & 0.209 & 0.643 \\
\hline & [OTRODIAG=0] & 0.821 & 0.3722 & 0.091 & 1.55 & 4.863 & 1 & 0.027 & 2.272 & 1.096 & 4.712 \\
\hline $\mathrm{AR}(1)$ & [OTRODIAG=0] & 0.858 & 0.3085 & 0.254 & 1.463 & 7.744 & 1 & 0.005 & 2.359 & 1.289 & 4.319 \\
\hline
\end{tabular}

*Se muestra solo los resultados significativos

De lo mostrado en la Tabla 1, se desprende que los mejores predictores para explicar el comportamiento del segmento ST son el sexo y otro diagnóstico adicional al IAM, ya que en todos los casos se llegó a la convergencia en la estimación de los parámetros cuando la matriz de correlaciones de trabajo tiene estructura independiente; en los demás casos no se llegó a alcanzar la convergencia. Se observa que los hombres parecen retardar su recuperación, en comparación con las mujeres que han sufrido IAM, con un $\mathrm{OR}=0.367$ cuando la estructura de la correlación es independiente; en los demás casos no hay diferencias. Para los pacientes que tienen alguna pato- logía adicional, a diferencia de los que han sufrido solamente IAM, demoran más en recuperarse, donde se tiene un $\mathrm{OR}=2.272$ cuando la estructura de la correlación es independiente y un $\mathrm{OR}=2.359$ cuando la estructura de la correlación es AR(1). Se muestra que ambos predictores son relevantes para explicar el comportamiento del segmento ST. Se confirma la significancia del factor otro diagnóstico, pareciendo ser el factor más importante para explicar la evolución de este segmento.

Aquí se tomó como variable respuesta la evolución de la onda T (dicotómica: normal o anormal) y como predictores al diagnóstico, otro diagnóstico, sexo y edad del paciente con 
IAM. Se procesaron los datos teniendo en cuenta las distintas matrices de trabajo. En primer lugar, tomando la de independencia; en segundo lugar, la AR(1); luego la intercambiable y, por último, la matriz de trabajo sin estructura. Los resultados significativos se muestran a continuación:

\section{Tabla 2}

Estimación de parámetros con tipo de respuesta logística ordinal, función de enlace logit acumulado, con modelo aditivo de efectos principales y con distintas estructuras de correlaciones tomando como variable respuesta la Onda T

\begin{tabular}{|c|c|c|c|c|c|c|c|c|c|c|c|}
\hline \multirow{2}{*}{$\begin{array}{l}\text { Estructura de } \\
\text { la correlación }\end{array}$} & \multirow{2}{*}{$\begin{array}{l}\text { Pará- } \\
\text { metro }\end{array}$} & \multirow{2}{*}{ B } & \multirow{2}{*}{$\begin{array}{l}\text { Típ. } \\
\text { Error }\end{array}$} & \multicolumn{2}{|c|}{$\begin{array}{c}\text { Intervalo de } \\
\text { confianza de } \\
\text { Wald } 95 \%\end{array}$} & \multicolumn{3}{|c|}{$\begin{array}{c}\text { Contraste de } \\
\text { hipótesis }\end{array}$} & \multirow{2}{*}{$\begin{array}{l}\text { Exp } \\
\text { (B) }\end{array}$} & \multicolumn{2}{|c|}{$\begin{array}{c}\text { Intervalo de } \\
\text { confianza } \\
\text { de Wald de } \\
\operatorname{Exp}(\text { B) } 95 \%\end{array}$} \\
\hline & & & & $\begin{array}{l}\text { Infe- } \\
\text { rior }\end{array}$ & $\begin{array}{l}\text { Supe- } \\
\text { rior }\end{array}$ & $\begin{array}{l}\text { Chi- } \\
\text { cuadra- } \\
\text { do de } \\
\text { Wald }\end{array}$ & G1 & Sig. & & $\begin{array}{l}\text { Infe- } \\
\text { rior }\end{array}$ & $\begin{array}{l}\text { Supe } \\
\text { rior }\end{array}$ \\
\hline Intercambiable & [SEXO=1] & 2.291 & 0.9832 & 0.364 & 4.219 & 5.432 & 1 & 0.02 & 9.89 & 1.44 & 67.933 \\
\hline
\end{tabular}

*Se muestra solo los resultados significativos

De la Tabla 2 se desprende que el predictor sexo solo tiene relevancia para describir la evolución de la onda T del paciente con IAM, cuando la estructura de la correlación es intercambiable con un OR=9.89.

\section{DISCUSIÓN}

Este estudio es el primero en el Perú donde se utilizan los modelos GEE aplicado a la evolución del IAM. Casi la totalidad de los pacientes viven en Lima Metropolitana.

De los 15 pacientes analizados, predomina el sexo masculino (80\%) sobre el femenino $(20 \%)$; situación similar a la descrita en el registro publicado de síndrome coronario agudo (SCA) del Hospital Loayza de Lima, Perú, así como en el multinacional es- tudio GRACE y en el registro chileno de angina inestable; aunque en este último, la población masculina es algo menor, cercana al 60\% (Carrión, Becerra, Pinto, \& Postigo, 2007).

De los pacientes 53\% tuvieron solo IAM y, el otro $47 \%$, fue diagnosticado con IAM no $Q$, lo cual indica que no hay diferencia significativa en los porcentajes de ambos diagnósticos.

En relación a la edad de presentación del IAM se encuentra el promedio alrededor de 64 años, no habiendo diferencias significativas con respecto al sexo, a diferencia de la situación que se aprecia en el registro chileno de angina inestable, donde las mujeres aumentan su incidencia a edades mayores de 60 a 70 años (Corbalan, y otros, 2004). 
Por los hallazgos encontrados, los factores analizados no explican significativamente la evolución del complejo QRS del ECG de los pacientes con IAM, como era de esperar.

\section{CONCLUSIONES Y RECOMEN- DACIONES}

Se probó que el sexo y otro diagnóstico son relevantes para describir la evolución del segmento ST de los electrocardiogramas registrados a los pacientes con IAM.

La edad y el tipo de diagnóstico (IAM o IAM no Q), no fueron relevantes para explicar la evolución del segmento ST de los pacientes con IAM. Para el modelo que toma como variable respuesta la evolución del segmento ST, y como predictores el sexo y otros diagnósticos, resultó como mejor matriz de correlaciones de trabajo la independiente. De lo anterior se desprende que el resultado del ECG no está determinado significativamente por los anteriores, como contrariamente se pudiera pensar. Los modelos GEE tienen una gran flexibilidad para el tratamiento de los distintos tipos de variable respuesta y, adicionalmente, tienen la facilidad de trabajar con datos correlacionados o medidas repetidas como en el presente estudio.

Se sugiere informatizar la información, ya que es bastante dificultoso determinar la evolución del paciente de las historias clinicas a causa de que no se registran las fechas en muchos casos, así como se producen muchas pérdidas de documentación de las historias clínicas. De acuerdo a estos resultados, se debería tomar en cuenta la diferenciación de los tratamientos para los pacientes de distintos sexos y distintas morbilidades. Se deberian incluir más predictores para tratar de explicar mejor el comportamiento de los indicadores de evolución en los pacientes con IAM, siendo esto posible si hubiese más orden y legibilidad en la construcción de las historias clínicas. Necesariamente se deben de utilizar los modelos GEE cuando existe correlación entre los datos. 


\section{REFERENCIAS BIBLIOGRÁFICAS}

Agresti, A. (2007). An Introduction to Categorical Data Analysis. New York: John Wiley \& Sons, Inc.

Bermudez, F. (1998). Electrocardiografía diagnóstica. Caracas: McGraw_Hill Interamericana de Venezuela, S. A.

Bobashev, G. V., \& Anthony, J. C. (2000). Use of Alternating Logistic Regression in Studies of Drug Use Clustering. Substance Use and Misuse, 245-267.

Carrión, M., Becerra, L., Pinto, J., \& Postigo, R. (2007). Características Clinicas y seguimiento a 30 dias de pacientes con sindrome isquémico. Revista de la Sociedad Peruana de Medicina Interna, 53-59.

Corbalan, R., Nazzal, C., Eggers, G., Bertolucci, J., Prieto, J., \& col. (2004). Resultados del primer registro chileno de angina inestable: característica clínicas, perfil de riesgo y tratamiento. Revista Médica de Chile, 135-143.

Jenkins, D., \& Gerred, S. (2012). ECG en ejemplo. Barcelona: Elsevier España, S. L.

Liang, K., \& Zeger, S. (1986). Longitudinal data analysis using generalized linear models. Biometrika, 13-22. 